\title{
Hypoglycemic effects of aqueous persimmon leaf extract in a murine model of diabetes
}

\author{
UI-JIN BAE $^{1 *}$, SOO-HYUN PARK ${ }^{2 *}$, SU-YOUNG JUNG ${ }^{2}$, BYUNG-HYUN PARK ${ }^{1}$ and SOO-WAN CHAE ${ }^{2,3}$ \\ ${ }^{1}$ Department of Biochemistry, Chonbuk National University Medical School, Deokjin-gu, Jeonju, Jeonbuk 561-756; \\ ${ }^{2}$ Clinical Trial Center for Functional Foods, Chonbuk National University Hospital, Deokjin-gu, Jeonju, Jeonbuk 561-712; \\ ${ }^{3}$ Department of Pharmacology, Chonbuk National University Medical School, \\ Deokjin-gu, Jeonju, Jeonbuk 561-756, Republic of Korea
}

Received August 9, 2014; Accepted April 15, 2015

DOI: $10.3892 / \mathrm{mmr} .2015 .3766$

\begin{abstract}
Previously, powdered persimmon leaves have been reported to have glucose- and lipid-lowering effects in diabetic $(\mathrm{db} / \mathrm{db})$ mice. As persimmon leaf is commonly consumed as tea, an aqueous extract of persimmon leaves (PLE) was prepared and its anti-diabetic efficacy was investigated. In the present study, PLE was tested for its inhibitory activity on $\alpha$-glucosidase in vitro. An oral maltose tolerance test was performed in diabetic mice. Next, the acute effect of PLE was examined in streptozotocin-induced diabetic mice. Last, the long-term effect of PLE supplementation was assessed in $d b / d b$ after eight weeks. An oral glucose tolerance test, biochemical parameters, as well as histological analyses of liver and pancreas were evaluated at the end of the study. PLE inhibited $\alpha$-glucosidase activity and increased antioxidant capacity. Streptozotocin-induced diabetic mice pre-treated with PLE displayed hypoglycemic activity. Daily oral supplementation with PLE for eight weeks reduced body weight gain without affecting food intake, enhanced the glucose tolerance during the oral glucose tolerance test (OGTT), improved blood lipid parameters, suppressed fat accumulation in the liver and maintained islet structure in $d b / d b$ mice. Further mechanistic study showed that PLE protected pancreatic islets from glucotoxicity. In conclusion, the results of the present study
\end{abstract}

Correspondence to: Professor Soo-Wan Chae, Department of Pharmacology, Chonbuk National University Medical School, 567 Baekje-daero, Deokjin-gu, Jeonju, Jeonbuk 561-756, Republic of Korea

E-mail: soowan@jbnu.ac.kr

Professor Byung-Hyun Park, Department of Biochemistry, Chonbuk National University Medical School, 567 Baekje-daero, Deokjin-gu, Jeonju, Jeonbuk 561-756, Republic of Korea

E-mail: bhpark@jbnu.ac.kr

*Contributed equally

Key words: water extract of persimmon leaves, $\alpha$-glucosidase, $\beta$-cells, streptozotocin, $d b / d b$ indicated that PLE exhibits considerable anti-diabetic effects through $\alpha$-glucosidase inhibition and through the maintenance of functional $\beta$-cells. These results provided a rationale for the use of persimmon leaf tea for the maintenance of normal blood glucose levels in diabetic patients.

\section{Introduction}

The prevalence of diabetes in Korean patients has dramatically increased from 1.5 to $9.9 \%$ over the last 40 years (1). It is anticipated that the prevalence of diabetes will rise to $11.4 \%$ by 2030 . This drastic increase in diabetic patients is ultimately associated with secondary micro- and macro-vascular complications (2). Therefore, effective approaches to control blood glucose are required to prevent vascular complications and improve the quality of life of diabetic patients. Initial management often involves lifestyle interventions, including diet and exercise, but in most cases, pharmacotherapy is also required as the disease progresses (2). However, medications used to control blood glucose often cause metabolic side effects, including weight gain and organ toxicity $(3,4)$. Thus, development of alternative therapies is of paramount importance, and natural products that can manage patients' blood glucose levels without noticeable side effects are gaining considerable attention.

Persimmon (Diospyros kaki), a fruit tree that is native to China and belongs to the family of Ebenaceae, is widely distributed in eastern Asia. The fruit of the persimmon tree is consumed as food, whereas the young leaf is mainly used for tea. Persimmon leaf tea contains several bioactive compounds, including flavonoids, triterpenoids, tannins and carotenoids (5-9). A number of studies have reported the beneficial effects of persimmon leaf extract (PLE) on hypertension (5), stroke (10), atherosclerosis (11) and dermatitis (12). Recently, Jung et al (13) investigated the metabolic effects of PLE using type 2 diabetic $(d b / d b)$ mice. After oral administration of powdered persimmon leaves for five weeks, glucose- and lipid-lowering effects were observed in the animals, which also led to amelioration of hyperglycemia, dyslipidemia and fatty liver.

In the present study, the anti-diabetic efficacy of PLE in streptozotocin-induced diabetic mice and $d b / d b$ mice 
was investigated. Furthermore, the underlying mechanism of the anti-diabetic effect of PLE was investigated, particularly focusing on $\alpha$-glucosidase inhibition and pancreatic $\beta$-cell-protecting activities.

\section{Materials and methods}

Reagents. Unless otherwise stated, all reagents were purchased from Sigma-Aldrich (St. Louis, MO, USA). Collagenase was purchased from Roche Diagnostics (Indianapolis, IN, USA).

Preparation of PLE. Persimmon leaves were raised and harvested in Wanju (Jeonbuk, Korea) in June 2013 by Dongsangmyeon Saramdeul Inc. (Jeonbuk, Korea). Persimmon leaves were dried in the shade for one week prior to being powdered and passed through 60 -mesh sieves. One volume of persimmon leaf powder was added to 10 volumes of distilled water and extracted at $90-100^{\circ} \mathrm{C}$ for $3 \mathrm{~h}$. The aqueous phase was filtered and concentrated with a vacuum evaporator (Eyela, Japan). After lyophilization, the powder was stored at $-80^{\circ} \mathrm{C}$ until used. The components of PLE were analyzed by the Development Institute of Traditional Korean Medicine (Jeonnam, Korea) using a high-pressure liquid chromatography workstation (Shimadzu, Japan) (Fig. 1). Analyses were performed on an X-bridged C18 column with a mobile phase gradient of A) $0.1 \%$ formic acid and B) acetonitrile over $50 \mathrm{~min}$. Gradient elution was programmed at a flow rate of $0.25 \mathrm{ml} / \mathrm{min}$ as follows: $0 \mathrm{~min}(100 \%), 10 \mathrm{~min}(90 \%), 30 \mathrm{~min}$ (40\%), $45 \mathrm{~min}(30 \%)$ and $50 \mathrm{~min}(90 \%)$. The injection volume was $20 \mu \mathrm{l}$. The column temperature was kept constant at $25^{\circ} \mathrm{C}$, and the mobile phase flow rate was $1 \mathrm{ml} / \mathrm{min}$ with ultraviolet detection at $265 \mathrm{~nm}$. PLE was standardized to contain 4-7 mg total quercetin 3-O-2'galloylglucoside $\left(\mathrm{C}_{24} \mathrm{H}_{24} \mathrm{O}_{19}\right)$ and kaempferol 3-O-2'galloylglucoside $\left(\mathrm{C}_{24} \mathrm{H}_{24} \mathrm{O}_{18}\right)$ per $1 \mathrm{~g}$ of extract.

In vitro $\alpha$-glucosidase assay. Yeast $\alpha$-glucosidase $(0.5 \mathrm{U})$ dissolved in $0.2 \mathrm{M}$ potassium phosphate buffer ( $\mathrm{pH}$ 6.8) was mixed with various concentrations of PLE or acarbose. After incubation at $37^{\circ} \mathrm{C}$ for $15 \mathrm{~min}, 3 \mathrm{mM} p$-nitrophenyl- $\alpha$-D-gluc opyranoside was added. The reaction was further incubated at $37^{\circ} \mathrm{C}$ for $10 \mathrm{~min}$ and then stopped by the addition of $0.1 \mathrm{M}$ $\mathrm{Na}_{2} \mathrm{CO}_{3}$. The absorption (Abs) of 4-nitrophenol was measured at $405 \mathrm{~nm}$. Reaction mixture without any sample was used as a control, and the mixture without substrate was used as a blank. The percent inhibition of $\alpha$-glucosidase was calculated as [1-( $\left.\left.\mathrm{Abs}_{\text {sample }}-\mathrm{Abs}_{\text {blank }}\right) / \mathrm{Abs}_{\text {control }}\right] \times 100$. Measurements were performed in triplicate.

Oxygen free radical scavenging assay. The anti-oxidant activity of each sample extract was assessed by the ability of the extract to scavenge 2,2-diphenyl-1-picrylhydrazyl (DPPH) free radicals. The extracts, in separate test tubes, were allowed to react with DPPH. DPPH free radical scavenging activity was monitored by measuring the decline in absorbance at $517 \mathrm{~nm}$. Butylated hydroxyanisole was used as the standard compound.

Experimental design. Pathogen-free, male C57BL/6 mice were purchased from Orientbio (Sungnam, Korea). The mice were housed at $20^{\circ} \mathrm{C}$ with $50 \%$ relative humidity, a 12-h light/dark cycle (light from 6:00 am to 6:00 pm) and were provided free access to drinking water. To induce diabetes, eight-week-old male C57BL/6 mice were injected via the tail vein with $100 \mathrm{mg} / \mathrm{kg}$ body weight streptozotocin (STZ) dissolved in $0.1 \mathrm{~mol} / 1$ sodium citrate buffer $(\mathrm{pH} 4.0)$. The control mice received citrate buffer alone. PLE (50 or $250 \mathrm{mg} / \mathrm{kg}$ body weight) was injected daily for five days via oral gavage prior to administration of STZ. STZ was first administered on day one. On day six, mice were sacrificed by decapitation without anesthesia and trunk blood was collected.

In addition, seven-week-old male C57BL/KsJ- $d b / d b$ $(d b / d b)$ mice were purchased from the Jackson Lab (Bar Harbor, ME, USA) and fed a normal chow diet. Starting at eight weeks of age, the point at which the mice become diabetic, the $d b / d b$ mice were treated with PLE (50 or $250 \mathrm{mg} / \mathrm{kg}$ ) for eight weeks via oral gavage once daily. Each group was made up of five mice. As a positive control, acarbose $(10 \mathrm{mg} / \mathrm{kg})$ was administered instead of PLE. Food consumption and body weight were recorded every week. At the end of the experimental period, an oral glucose tolerance test (OGTT; $1 \mathrm{~g} / \mathrm{kg}$ body weight) was performed. After a $14 \mathrm{~h}$ fast, glucose was administered by oral gavage $(2 \mathrm{mg} / \mathrm{g})$. The blood glucose level was subsequently determined from the tail vein at $0,15,30,60$ and 120 min following the glucose administration. Animals were sacrificed by decapitation, after which blood samples were collected, and livers were removed and weighed. All of the animal experiments were performed in accordance with the Guide for the Care and Use of Laboratory Animals published by the US National Institutes of Health (NIH Publication no. 85-23, revised 2011). The protocol of the present study was approved by the Institutional Animal Care and Use Committee of Chonbuk National University (permit no. CBU 2014-00048).

Oral maltose tolerance test in streptozotocin-induced diabetic mice. Mice were classified into four groups (1-4) containing five mice each. Groups 1 and 2 received phosphate-buffered saline (PBS) as a negative control or acarbose $(3 \mathrm{mg} / \mathrm{kg}$ ) as a positive control, respectively. Groups 3 and 4 were treated with PLE at two doses $(50$ and $250 \mathrm{mg} / \mathrm{kg})$. All samples were administered orally to 12 -h fasted mice, and $3 \mathrm{~g} / \mathrm{kg}$ of maltose was administered $5 \mathrm{~min}$ thereafter. Blood was collected from the tail vein at $0,15,30,60$ and $120 \mathrm{~min}$ after loading maltose.

Biochemical analyses. Blood glucose levels were measured by Accu-Chek Aviva glucose monitors (Roche Diagnostics, Indianapolis, IN, USA) and plasma insulin was measured using an ELISA kit (cat. no. EZRMI-13K; Millipore, Bedford, MA, USA). Plasma levels of total cholesterol (TC), triglyceride (TG) and HDL-cholesterol were measured using commercially available kits (cat. no's. AM202-K, AM157S-K and AM203-K, respectively; Asan Pharmaceutical, Seoul, Korea). For liver TG quantification, liver tissues were homogenized and extracted in chloroform, methanol and DW (2/1/1 ratio).

Histology. Tissues were removed and immediately placed in $10 \%$ formalin solution, embedded in paraffin and cut into $5-\mu \mathrm{m}$ sections. Specimens were stained with 


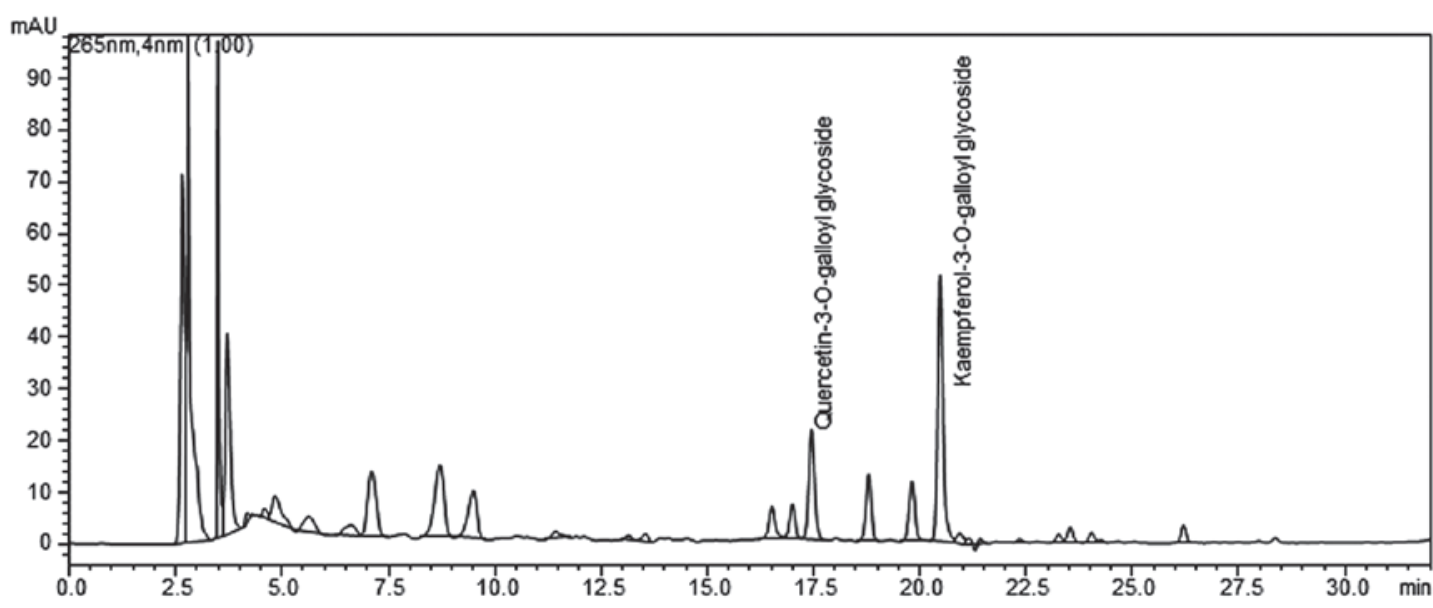

Figure 1. High-performance liquid chromatogram of persimmon leaf extract, which was standardized to contain $7.5 \mathrm{mg}$ quercetin 3-O-2'galloylglucoside (and kaempferol 3-O-2'galloylglucoside per $1 \mathrm{~g}$ extract. Analyses were performed on an X-bridged C18 column with a mobile phase gradient of A) $0.1 \%$ formic acid and B) acetonitrile over $50 \mathrm{~min}$. The injection volume was $20 \mu \mathrm{l}$. The column temperature was kept constant at $25^{\circ} \mathrm{C}$, and the mobile phase flow rate was $1 \mathrm{ml} / \mathrm{min}$ with ultraviolet detection at $265 \mathrm{~nm}$.

hematoxylin and eosin (H\&E) to identify morphological changes. For immunohistochemical analysis, tissue sections were subjected to a microwave antigen retrieval procedure (1,000 watts for $5 \mathrm{~min}$; CPC-600; Cuisinart, East Windsor, $\mathrm{NJ}$, USA) in $0.01 \mathrm{~mol} / 1$ sodium citrate buffer. After blocking endogenous peroxidase, the sections were incubated with Protein Block Serum-Free (DAKO, Glostrup, Denmark) to block non-specific staining and then with rabbit anti-insulin antibody (cat. no. sc-9168; 1:100; Santa Cruz Biotechnology, Dallas, TX, USA) for $12 \mathrm{~h}$ at $4^{\circ} \mathrm{C}$. Peroxidase activity was detected using 3-amino-9-ethyl carbazole. Tissue sections were observed using a light microscope (Eclipse E600 polarizing microscope; Nikon, Tokyo, Japan).

Islet isolation and glucose-stimulated insulin secretion (GSIS) assay. Pancreatic islets were isolated from 12-weekold mice using the collagenase digestion method as previously described (14). Following isolation, islets were cultured overnight in RPMI-1640 supplemented with $2 \mathrm{mM}$ L-glutamine, $10 \%$ heat-inactivated fetal calf serum, 100 units $/ \mathrm{ml}$ penicillin and $100 \mu \mathrm{g} / \mathrm{ml}$ streptomycin in humidified air containing $5 \%$ $\mathrm{CO}_{2}$ at $37^{\circ} \mathrm{C}$. Prior to experiments, islets were washed three times in RPMI-1640 and cultured overnight. After the initial culture period, islets were cultured for three days in identical RPMI-1640 containing 5.5 or $30 \mathrm{mmol} / 1$ glucose and subsequently washed three times in Krebs-Ringer bicarbonate buffer [25 mM 4-(2-hydroxyethyl)-1-piperazineethanesulfonic acid, $115 \mathrm{mmol} / 1 \mathrm{NaCl}, 24 \mathrm{mmol} / 1 \mathrm{NaHCO}_{3}$, $5 \mathrm{mmol} / \mathrm{l} \mathrm{KCl}, 1 \mathrm{mmol} / 1 \mathrm{MgCl}_{2}, 2.5 \mathrm{mmol} / 1 \mathrm{CaCl}_{2}$ and $0.1 \%$ bovine serum albumin, $\mathrm{pH} 7.4$ ] containing $2.8 \mathrm{mmol} / 1$ D-glucose. Insulin secretion assays were performed with 2.8 or $16.7 \mathrm{mmol} / \mathrm{l}$ glucose and measured using an ELISA kit (cat. no. EZRMI-13K; Millipore, Bedford, MA, USA).

Statistical analysis. Statistical analysis was performed using analysis of variance and Duncan's tests on through GraphPad Prism v5.02 (GraphPad Software Inc., La Jolla, CA, USA). $\mathrm{P}<0.05$ was considered to indicate a statistically significant difference between values.

\section{Results}

PLE inhibits $\alpha$-glucosidase activity. The in vitro inhibitory activity of PLE against yeast $\alpha$-glucosidase is shown in Fig. 2A. PLE inhibited $\alpha$-glucosidase activity in a dose-dependent manner and therefore should be considered an effective $\alpha$-glucosidase inhibitor: PLE at a concentration of $100 \mu \mathrm{g} / \mathrm{ml}$ inhibited $\alpha$-glucosidase activity by $70.5 \%$ and was $16.0 \%$ less potent than acarbose, which was used as a positive control. The half maximal inhibitory concentration $\left(\mathrm{IC}_{50}\right)$ value on $\alpha$-glucosidase activity was $4.7 \mu \mathrm{g} / \mathrm{ml}$.

The results of the maltose tolerance experiment are presented in Fig. 2B. After the administration of maltose, the blood glucose levels in normal mice increased after $15 \mathrm{~min}$. This elevation was statistically significant compared with the levels at time 0 for each group. At $120 \mathrm{~min}$, the blood glucose levels returned to their basal values. Oral administration of $250 \mathrm{mg} / \mathrm{kg}$ PLE inhibited the increases in glucose levels after $30 \mathrm{~min}$, which was statistically significant compared with levels in the corresponding controls at each time-point. The administration of acarbose also significantly decreased the increase of postprandial blood glucose. The areas under the curve (AUC) for glucose were reduced by $15.34 \%$ by PLE at $50 \mathrm{mg} / \mathrm{kg}, 23.63 \%$ by PLE at $250 \mathrm{mg} / \mathrm{kg}$ and $26.51 \%$ by acarbose.

Furthermore, the anti-oxidant properties of PLE were determined using the cell-free DPPH assay. PLE inhibited DPPH activity in a dose-dependent manner (Fig. 3), which confirms the findings of a previous study (15).

PLE protects mice against STZ-induced diabetes. Powdered persimmon leaf has previously been reported to have glucoseand lipid-lowering effects in $d b / d b$ mice (13). First, the present study evaluated the hypoglycemic effects of PLE at concentrations of $50 \mathrm{mg} / \mathrm{kg}$ (low-dose) and $250 \mathrm{mg} / \mathrm{kg}$ (high-dose) in STZ-induced diabetic mice. As shown in Fig. 4A, a single intravenous injection of STZ induced the increase of fasting blood glucose levels to $\sim 400 \mathrm{mg} / \mathrm{dl}$. However, the fasting glucose levels were significantly reduced to $176 \mathrm{mg} / \mathrm{dl}$ in 
A

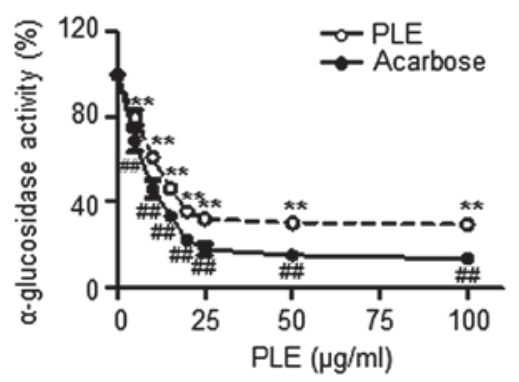

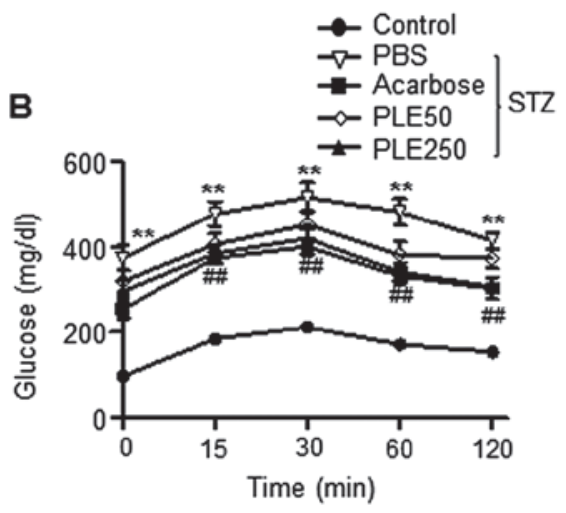

STZ

Figure 2. $\alpha$-Glucosidase inhibitory activity of PLE. (A) Effects of different concentrations of PLE on $\alpha$-glucosidase activity in vitro $\left(^{* *} \mathrm{P}<0.01,{ }^{\# \#} \mathrm{P}<0.01\right.$, vs. no treatment). (B) Effects of PLE on blood glucose levels after administration of $3 \mathrm{~g} / \mathrm{kg}$ maltose in STZ-induced diabetic mice. Values are expressed as the mean \pm standard error of the mean $\left(\mathrm{n}=5 ;{ }^{* *} \mathrm{P}<0.01\right.$, vs. control mice; ${ }^{\#} \mathrm{P}<0.05,{ }^{\# \#} \mathrm{P}<0.01$, vs. PBS-treated STZ mice). PLE50, persimmon leaf extract $(50 \mathrm{mg} / \mathrm{kg})$; PLE250, persimmon leaf extract (250 mg/kg); STZ, streptozotocin; PBS, phosphate-buffered saline; AUC, area under curve.
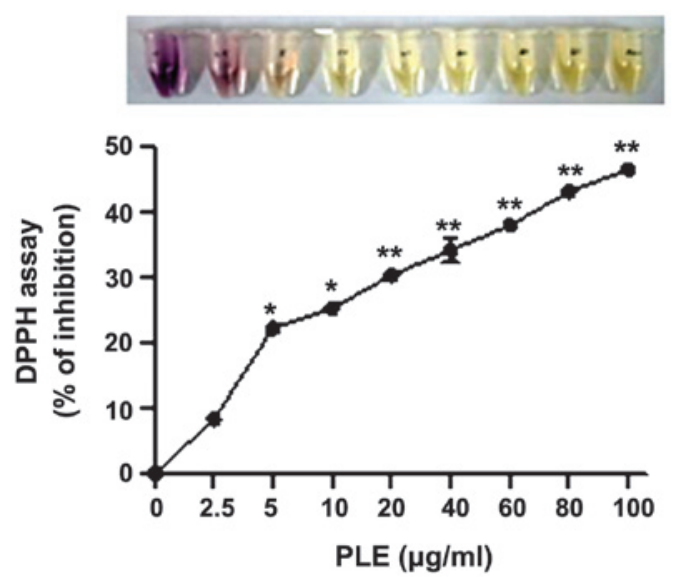

Figure 3. Free-radical scavenging activity of PLE using the cell-free DPPH assay. Values are expressed as the mean \pm standard error of the mean of three independent experiments $\left({ }^{*} \mathrm{P}<0.05,{ }^{* * *} \mathrm{P}<0.01\right.$, vs. no treatment). PLE, persimmon leaf extract; DPPH, 2,2-diphenyl-1-picrylhydrazyl radical.

the STZ mice pre-treated with high-dose PLE. The group of mice treated with acarbose $(10 \mathrm{mg} / \mathrm{kg})$ showed similar blood glucose levels. Of note, the body weight among the groups did not change over the course of the study (data not shown).

The effect of PLE on the post-prandial increase in blood glucose in STZ-induced diabetic mice was determined via OGTT. Consistent with the above results, oral administration of high-dose PLE significantly prevented an increase in plasma glucose levels (Fig. 4B). High-dose PLE and acarbose decreased the AUCs for the postprandial glucose responses by 61.6 and $62.4 \%$, respectively, compared with that in the PBS-treated group $(\mathrm{P}<0.01)$. These results indicated that treatment with PLE prevents STZ-induced $\beta$-cell damage in mice.

Long-term treatment with PLE ameliorates hyperglycemia and dyslipidemia in $\mathrm{db} / \mathrm{db}$ mice. To further evaluate the therapeutic effects of PLE on diabetes, the long-term anti-diabetic effects of PLE in $d b / d b$ mice were evaluated. Food intake was not significantly influenced by PLE treatment (Fig. 5A); however, the body weight was significantly decreased by high-dose PLE treatment (Fig. 5B). Diabetic $d b / d b$ mice at 16 weeks of age
A
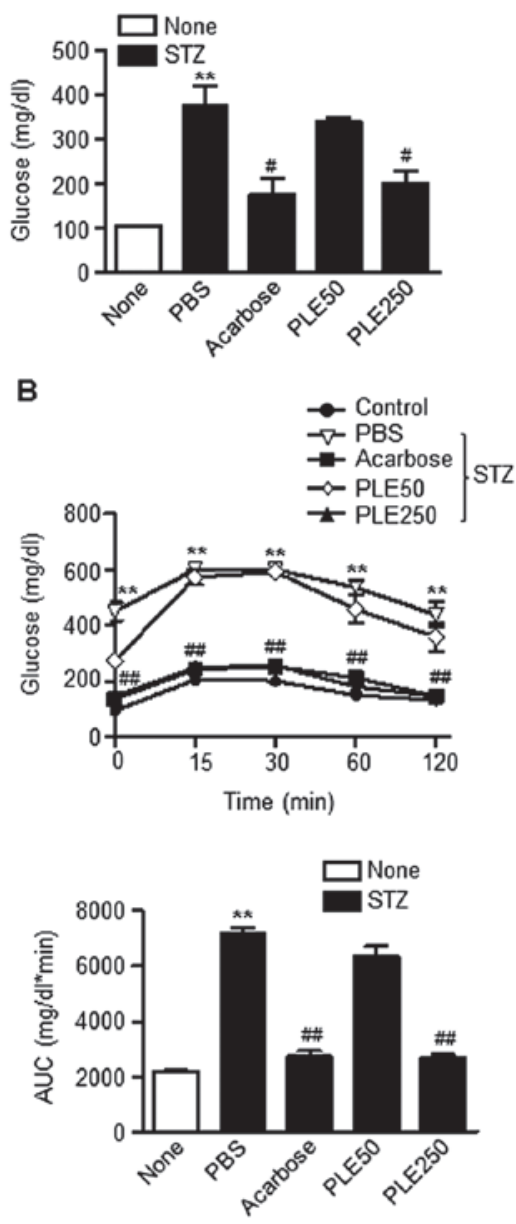

Figure 4. Improved glycemic control in STZ-induced hyperglycemic mice after short-term treatment with PLE. PLE50 or PLE250 was administered via oral gavage daily for five days prior to the administration of STZ. (A) Six days following STZ injection, levels of post-prandial glucose were determined. (B) Oral glucose tolerance tests - five days after STZ injection, glucose $(1 \mathrm{~g} / \mathrm{kg})$ was administered to overnight-fasted mice and plasma glucose concentrations were measured. The bar graph represents areas under the curve. Values are expressed as the mean \pm standard error of the mean $(\mathrm{n}=5) .{ }^{* *} \mathrm{P}<0.01$ vs. control mice; ${ }^{\#} \mathrm{P}<0.05,{ }^{\# \#} \mathrm{P}<0.01$ vs. PBS-treated STZ mice. PLE50, persimmon leaf extract $(50 \mathrm{mg} / \mathrm{kg})$; PLE250, persimmon leaf extract (250 mg/kg); STZ, streptozotocin; PBS, phosphate-buffered saline; AUC, area under curve. 

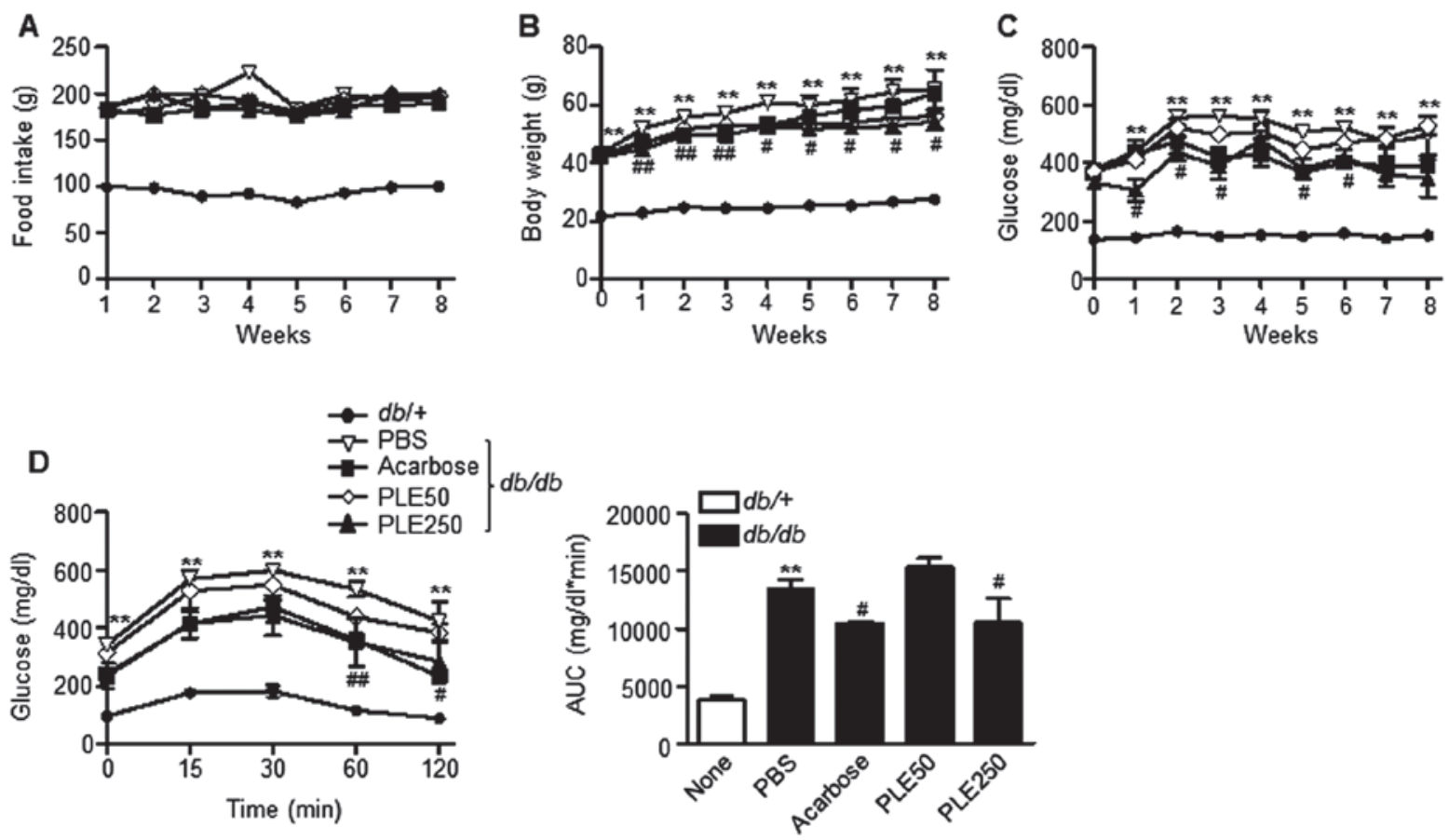

Figure 5. Improved glycemic control in $d b / d b$ mice after eight weeks of treatment with PLE. Mice received daily oral supplementation with PLE50 or PLE250 or $10 \mathrm{mg} / \mathrm{kg}$ acarbose for eight weeks. (A) Food intake and (B) body weight change were recorded at indicated times. (C) Post-prandial blood glucose levels. (D) Plasma glucose concentrations during oral glucose tolerance tests in overnight-fasted mice. The bar graph represents areas under the curve. Values are expressed as the mean \pm standard error of the mean $(\mathrm{n}=5)$. ${ }^{* *} \mathrm{P}<0.01$ vs. control mice; ${ }^{~} \mathrm{P}<0.05,{ }^{\# /} \mathrm{P}<0.01$ vs. PBS-treated $d b / d b$ mice. PLE50, persimmon leaf extract (50 mg/kg); PLE250, persimmon leaf extract ( $250 \mathrm{mg} / \mathrm{kg}$ ); PBS, phosphate-buffered saline; $d b / d b$, diabetic; AUC, area under curve.

that were fed normally had hyperglycemia with postprandial blood glucose levels of $\sim 494 \mathrm{mg} / \mathrm{dl}$ (Fig. 5C). High-dose PLE decreased postprandial blood glucose levels in $d b / d b$ mice as effectively as acarbose. The decrease in blood glucose levels by PLE appeared to be dose-dependent, as low doses of PLE $(50 \mathrm{mg} / \mathrm{kg})$ revealed no glucose lowering effects, whereas high doses of PLE $(250 \mathrm{mg} / \mathrm{kg})$ significantly decreases glucose levels $(\mathrm{P}<0.01)$.

An OGTT was performed to determine the effects of PLE on glucose tolerance after eight weeks of PLE treatment (Fig. 5D). The AUC for glucose response of high-dose PLE-treated $d b / d b$ mice was significantly lower than that of the $(d b / d b)$ mice. By contrast, oral administration of low-dose PLE did not show any improvement in glucose tolerance.

Plasma insulin levels between $d b / d b$ mice that received PBS and those that had been administered PLE were also compared. The serum insulin levels in high-dose PLE-treated mice tended to be higher than those in PBS-treated mice (Fig. 6A). In addition, as shown in Fig. 6B and C, high-dose PLE significantly lowered plasma TG $(160.5 \pm 15.9 \mathrm{mg} / \mathrm{dl} ; \mathrm{P}<0.01)$ and TC levels $(209.2 \pm 74.5 \mathrm{mg} / \mathrm{dl} ; \mathrm{P}<0.05)$ compared with those in the control mice $(208.9 \pm 32.4$ and $263.9 \pm 19.3 \mathrm{mg} / \mathrm{dl}$, respectively). Plasma HDL-cholesterol levels were not significantly different among groups (Fig. 6D). Overall, these results suggested that PLE has glucose- and lipid-lowering effects in $d b / d b$ mice.

Long-term treatment with PLE prevents fatty liver development in $\mathrm{db} / \mathrm{db}$ mice. As diabetes can trigger hepatic steatosis, livers were assessed for the extent of fat accumulation. The results indicated that PLE supplementation was associated with less steatotic livers (data not shown). Examination of
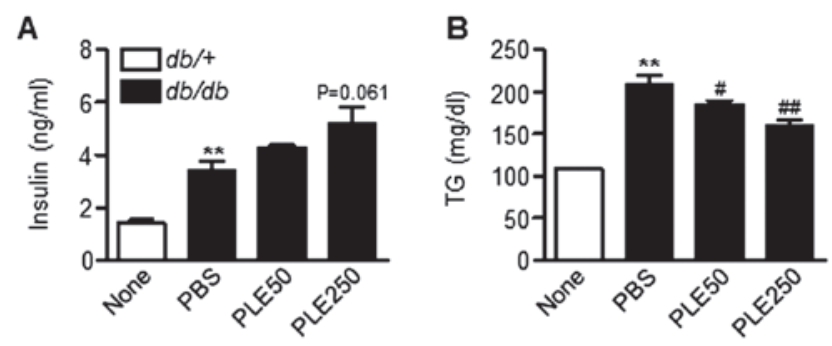

C

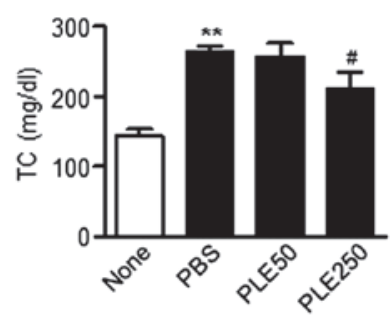

D

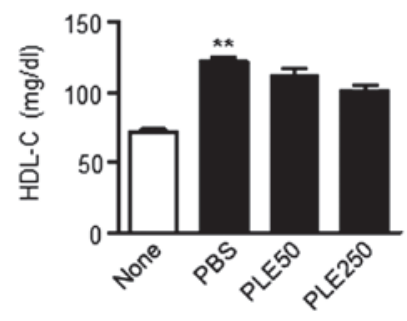

Figure 6. Biochemical parameters in $d b / d b$ mice after eight weeks of treatment with persimmon leaf extract. Mice received daily oral supplementation with PLE50 or PLE250. At the end of the study, plasma concentrations of (A) insulin, (B) TG, (C) TC and (D) HDL-C were determined. Values are expressed as the mean \pm standard error of the mean $(n=5) .{ }^{* *} \mathrm{P}<0.01$ vs. control mice; ${ }^{\#} \mathrm{P}<0.05,{ }^{\#} \mathrm{P}<0.01$ vs. PBS-treated $d b / d b$ mice. TG, triglyceride; TC, total cholesterol; HDL-C, high-density lipoprotein cholesterol; PLE50, persimmon leaf extract (50 mg/kg); PLE250, persimmon leaf extract (250 mg/kg); PBS, phosphate-buffered saline; $d b / d b$, diabetic.

H\&E-stained sections demonstrated marked macrovesicular steatosis in $d b / d b$ mice, and the degree of hepatic steatosis was markedly alleviated by PLE (Fig. 7A). Liver TG and liver weight were concordant with the histological findings 
A $d b / d b$

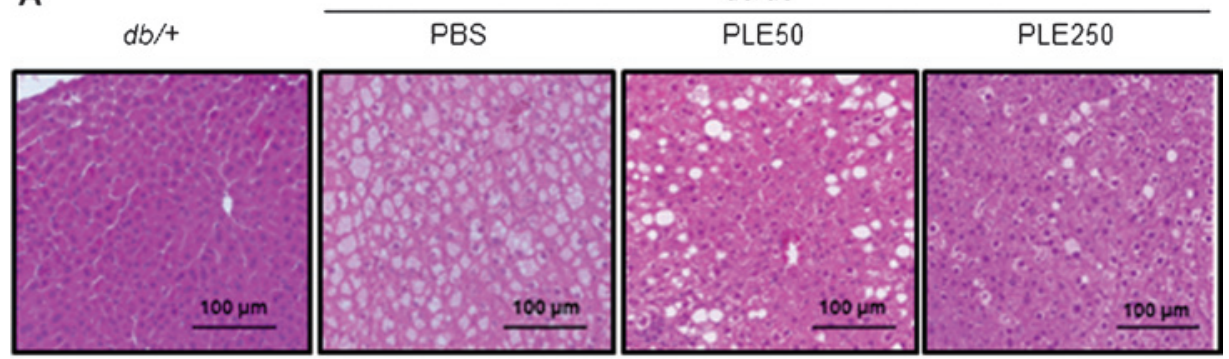

B
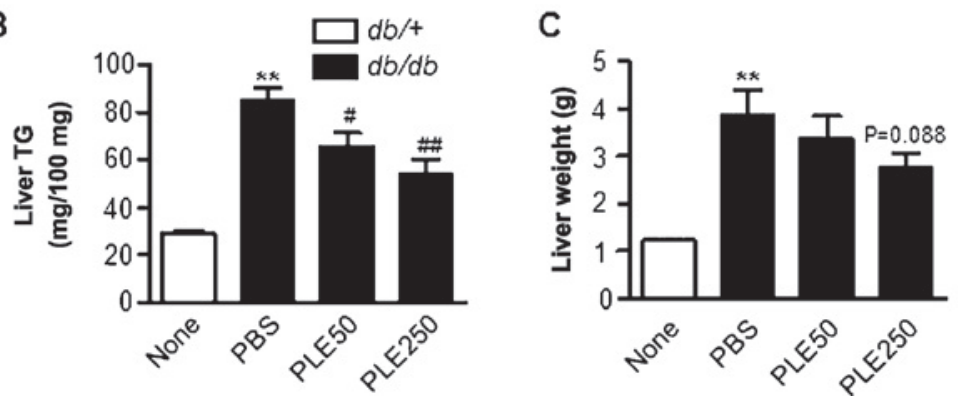

Figure 7. Prevention of liver steatosis in $d b / d b$ mice after eight weeks of treatment with PLE. (A) Representative hematoxylin and eosin-stained histological sections of liver from mice (magnification, $\mathrm{x} 200$ ). (B and C) Liver TG and wet weight were determined. Values are expressed as the mean \pm standard error of the mean ( $\mathrm{n}=5) .{ }^{* *} \mathrm{P}<0.01$ vs. control mice; ${ }^{\#} \mathrm{P}<0.05,{ }^{\# \#} \mathrm{P}<0.01$ vs. PBS-treated $d b / d b$ mice. PLE50, persimmon leaf extract $(50 \mathrm{mg} / \mathrm{kg})$; PLE250, persimmon leaf extract (250 mg/kg); PBS, phosphate-buffered saline; $d b / d b$, diabetic; TG, triglycerides.

A

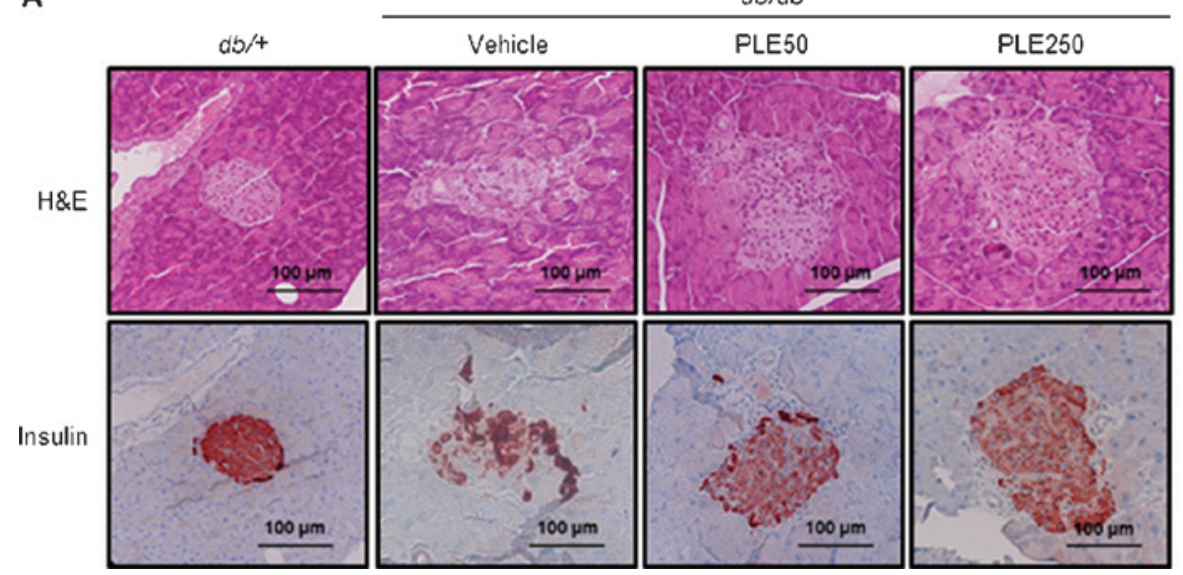

B

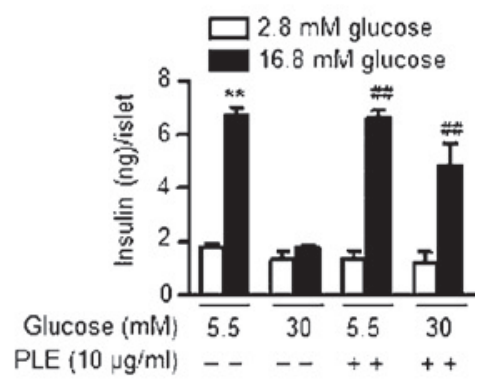

Figure 8. Prevention of pancreatic islet destruction in $d b / d b$ mice after eight weeks of treatment with PLE. (A) Sections of the pancreata of the mice were stained with H\&E or immunostained with insulin antibody (magnification, x200). (B) Mouse islets were treated with 5.5 or 30 mM glucose with or without PLE. Following a three-day incubation, insulin secretion assays were performed with 2.8 or $16.7 \mathrm{mmol} / 1 \mathrm{D}$-glucose. Values are expressed as the mean \pm standard error of the mean $(\mathrm{n}=5) .{ }^{* *} \mathrm{P}<0.01$ vs. control islets; ${ }^{*} \mathrm{P}<0.05,{ }^{\# \#} \mathrm{P}<0.01$ vs. $30 \mathrm{mM}$ glucose-incubated islets. H\&E, hematoxylin and eosin; PLE50, persimmon leaf extract (50 mg/kg); PLE250, persimmon leaf extract (250 mg/kg); PBS, phosphate-buffered saline; $d b / d b$, diabetic.

(Fig. 7B and C). Treatment of $d b / d b$ mice with high-dose PLE resulted in significant decreases in hepatic TG contents $(85.3 \pm 12.5 \mathrm{mg} / 100 \mathrm{mg}$ vs. $54.2 \pm 15.8 \mathrm{mg} / 100 \mathrm{mg} ; \mathrm{P}<0.01)$ and liver weight $(3.8 \pm 0.8$ vs. $2.7 \pm 0.6 \mathrm{~g} ; \mathrm{P}=0.088)$ as compared with that in the control $d b / d b$ mice.

Long-term treatment with PLE protects pancreatic $\beta$-cells in $d b / d b$ mice. As PLE administration increased plasma insulin levels (Fig. 6A), pancreata were examined using H\&E staining. Pancreatic islets of diabetic $d b / d b$ mice exhibited degeneration and poorly defined margins (Fig. 8A). Immunostaining with an insulin antibody showed weak insulin staining. By contrast, pancreatic islets of high-dose PLE-treated mice had a round shape and high insulin immunoreactivity, suggesting the protection of $\beta$-cells by PLE.

To determine the protective effects of PLE on $\beta$-cells, the present study examined whether it was effective in protecting pancreatic islets from glucotoxicity. Islets from mice were isolated and incubated under normal glucose $(5.5 \mathrm{mM})$ or high-glucose $(30 \mathrm{mM})$ culture conditions. Basal and glucose-stimulated insulin secretion was assessed after three days of culture (Fig. 8B). Results showed that the amount of glucose-stimulated insulin secretion was $6.65 \pm 0.48 \mathrm{ng} / \mathrm{islet} / \mathrm{h}$ in normal glucose-cultured islets and $4.80 \pm 1.48 \mathrm{ng} / \mathrm{islet} / \mathrm{h}$ in high-glucose-cultured islets. Following pre-treatment with PLE, however, the insulin secretion under high-glucose 
conditions was significantly restored to a level closer to the control value. Basal insulin release among the groups was similar. In conclusion, in vitro and in vivo results of the present study suggested that PLE exhibits protective effects on $\beta$-cells.

\section{Discussion}

In the present study, PLE was shown to improve the biochemical parameters of glucose and lipid metabolism and prevented fatty liver development in $d b / d b$ mice after eight weeks of oral supplementation. Similar results have been reported by a study of five-week treatment with powdered persimmon leaves (13), suggesting that long-term oral supplementation with persimmon leaf can effectively exert glycemic control in diabetic mice. In addition, five-day oral supplementation with PLE prevented diabetes development in STZ-treated mice. In recent meta-analysis studies, flavonoids have been reported to have acute and chronic effects on glucose and lipid metabolism $(16,17)$. As PLE contains a considerable amount of flavonoids, including quercetin and kaempferol (4), it is reasonable to expect PLE to exhibit acute as well as chronic anti-diabetic effects. However, even though PLE possesses hypoglycemic effects, the blood glucose levels in PLE-supplemented $d b / d b$ mice were still higher throughout the experimental period than those of wild-type $\mathrm{db} / \mathrm{m}$ mice, suggesting that PLE causes a certain improvement in glucose tolerance under hyperglycemic conditions.

Although the body weight decreased in $d b / d b$ mice after PLE supplementation, a similar decrease in food intake was not observed. This finding is in contrast with results from a study by Jung et al (13), in which oral administration of the powder of persimmon leaves caused significant decreases in body weight gain and food intake compared to those in control group mice. This discrepancy may result from a difference in persimmon leaf sources between the two studies. Furthermore, the present study used PLE, whereas Jung et al fed mice with $5 \%$ powder of persimmon leaves. As the aqueous extract and powdered persimmon leaves display differences in their chemical composition, their application is likely to have different outcomes. In the present study, the conclusion that PLE reduced body weight and/or improved glucose tolerance simply by reducing food intake can be excluded.

Of note, the hypoglycemic effect of PLE in $d b / d b$ mice was observable as early as one week after PLE supplementation. This finding led to the hypothesis that $\alpha$-glucosidase and $\alpha$-amylase inhibition are possible underlying mechanisms of the anti-diabetic effects of PLE, as these enzymes are involved in the digestion of complex carbohydrates from food into absorbable monosaccharides (18). Accordingly, an in vitro study was performed to examine the effects of PLE on $\alpha$-glucosidase activity. The results revealed that PLE inhibited $\alpha$-glucosidase activity in a dose-dependent manner with an $\mathrm{IC}_{50}$ value of $4.7 \mu \mathrm{g} / \mathrm{ml}$. In the following oral maltose tolerance test in normal mice, PLE showed marked $\alpha$-glucosidase inhibitory activity. In addition, a study by Kawakami et al (9) reported $\alpha$-amylase inhibitory activity of persimmon leaves. Therefore, inhibition of $\alpha$-glucosidase and/or $\alpha$-amylase by PLE may prolong overall digestion time, causing a delay in glucose absorption, consequently reducing the rapid increase of postprandial blood glucose.
PLE significantly suppressed the increase in the post-prandial blood glucose levels as compared to those in PBS-treated $d b / d b$ mice. Insulin has a pivotal role in maintaining the post-prandial glucose levels within a normal range by enhancing glycogen synthesis and glycolysis, and by suppressing gluconeogenesis (19). In general, the $d b / d b$ mice exhibited an initial phase of hyperinsulinemia to compensate for insulin resistance and progressively develop insulinopenia with age, a characteristic commonly observed in patients during late stages of type 2 diabetes (20). In $d b / d b$ mice supplemented with PLE, the plasma insulin levels were higher than those in PBS-treated $d b / d b$ mice. In addition, islet architecture was relatively well preserved and the mass of insulin-immunoreactive $\beta$-cells was increased in PLE-supplemented mice, suggesting that PLE-supplemented $d b / d b$ mice still displayed insulin-secreting $\beta$-cell masses. This assumption was evidenced by ex vivo experiments using isolated islets. Assessment of insulin secretion capacities after culturing of islets under glucotoxic conditions showed that glucose-stimulated insulin secretion was increased in PLE-treated islets as compared with that in untreated islets.

In conclusion, the present study provided further evidence for the anti-diabetic efficacy of PLE in STZ-induced diabetic mice and $d b / d b$ mice, which was comparable to the effect elicited by acarbose. Glucose tolerance during OGTT was enhanced, lipid parameters were improved and fat accumulation in the liver was suppressed in PLE-supplemented mice compared to those of the control mice. These beneficial effects are at least partially mediated via suppression of $\alpha$-glucosidase activity and preserved, functional $\beta$-cell masses. The former leads to decreased blood glucose levels and the latter leads to increased insulin levels. Therefore, the results of the present study implied that supplementing pre-diabetes or diabetes patients with PLE may be a way to maintain blood glucose levels within a normal range.

\section{Acknowledgements}

The present study was supported by a Regional Agri-Food Lead Cluster Promotion Project from Wanju-gun, Republic of Korea.

\section{References}

1. Kim DJ: The epidemiology of diabetes in Korea. Diabetes Metab J 35: 303-308, 2011.

2. Yamagishi $\mathrm{S}$ and Imaizumi T: Diabetic vascular complications: Pathophysiology, biochemical basis and potential therapeutic strategy. Curr Pharm Des 11: 2279-2299, 2005.

3. Derosa G and Maffioli P: Thiazolidinediones plus metformin association on body weight in patients with type 2 diabetes. Diabetes Res Clin Pract 91: 265-270, 2011.

4. Mitri J and Hamdy O: Diabetes medications and body weight. Expert Opin Drug Saf 8: 573-584, 2009.

5. Kawakami K, Shibukura Y, Kanno T, Furuki T, Aketa S and Hirayama M: Identification of 2'-galloylated flavonol 3-o-glycosides accumulating in developing leaves of persimmon. Phytochem Anal 22: 403-410, 2011.

6. Liu L, Liu RL, Zhang J and Zhang ZQ: Study on the PEG-based microwave-assisted extraction of flavonoid compounds from persimmon leaves. J Sep Sci 35: 3412-3420, 2012.

7. Chen G, Lu H, Wang C, et al: Effect of five triterpenoid compounds isolated from leaves of Diospyros kaki on stimulus-induced superoxide generation and tyrosyl phosphorylation in human polymorphonuclear leukocytes. Clin Chim Acta 320: 11-16, 2002. 
8. Chen G, Wang ZQ and Jia JM: Three minor novel triterpenoids from the leaves of Diospyros kaki. Chem Pharm Bull (Tokyo) 57: $532-535,2009$.

9. Kawakami K, Aketa S, Nakanami M, Iizuka S and Hirayama M: Major water-soluble polyphenols, proanthocyanidins, in leaves of persimmon (Diospyros kaki) and their alpha-amylase inhibitory activity. Biosci Biotechnol Biochem 74: 1380-1385, 2010.

10. Bei W, Peng W, Zang L, Xie Z, Hu D and Xu A: Neuroprotective effects of a standardized extract of Diospyros kaki leaves on MCAO transient focal cerebral ischemic rats and cultured neurons injured by glutamate or hypoxia. Planta Med 73: 636-643, 2007.

11. Katsube T, Tabata H, Ohta Y, et al: Screening for antioxidant activity in edible plant products: comparison of low-density lipoprotein oxidation assay, DPPH radical scavenging assay and Folin-Ciocalteu assay. J Agric Food Chem 52: 2391-2396, 2004.

12. Kotani M, Matsumoto M, Fujita A, et al: Persimmon leaf extract and astragalin inhibit development of dermatitis and IgE elevation in NC/Nga mice. J Allergy Clin Immunol 106: 159-166, 2000.

13. Jung UJ, Park YB, Kim SR and Choi MS: Supplementation of persimmon leaf ameliorates hyperglycemia, dyslipidemia and hepatic fat accumulation in type 2 diabetic mice. PLoS One 7: e49030, 2012.
14. Lee JH, Song MY, Song EK, et al: Overexpression of SIRT1 protects pancreatic beta-cells against cytokine toxicity by suppressing the nuclear factor- $\mathrm{\kappa} \mathrm{B}$ signaling pathway. Diabetes 58 : 344-351, 2009.

15. Sun L, Zhang J, Lu X, Zhang L and Zhang Y: Evaluation to the antioxidant activity of total flavonoids extract from persimmon (Diospyros kaki L.) leaves. Food Chem Toxicol 49: 2689-2696, 2011.

16. Liu YJ, Zhan J, Liu XL, Wang Y, Ji J and He QQ: Dietary flavonoids intake and risk of type 2 diabetes: A meta-analysis of prospective cohort studies. Clin Nutr 33: 59-63, 2014.

17. van Dam RM, Naidoo N and Landberg R: Dietary flavonoids and the development of type 2 diabetes and cardiovascular diseases: review of recent findings. Curr Opin Lipidol 24: 25-33, 2013.

18. Kumar S, Narwal S, Kumar V and Prakash O: $\alpha$-glucosidase inhibitors from plants: A natural approach to treat diabetes. Pharmacogn Rev 5: 19-29, 2011.

19. Bouché C, Serdy S, Kahn CR and Goldfine AB: The cellular fate of glucose and its relevance in type 2 diabetes. Endocr Rev 25: 807-830, 2004.

20. Kodama H, Fujita M and Yamaguchi I: Development of hyperglycaemia and insulin resistance in conscious genetically diabetic (C57BL/KsJ- $d b / d b$ ) mice. Diabetologia 37: 739-744, 1994. 